\title{
COnE)-(OES
}

CIENCIA E TECNOLOGIA

\section{AVALIAÇÃO DA PERCEPÇÃO QUANTO AO GERENCIAMENTO DOS RESÍDUOS SÓLIDOS EM ESTABELECIMENTOS VETERINÁRIOS DE SOBRAL/CE}

\author{
Francisco Amilcar Moreira Junior Amilcar Moreira ${ }^{1}$, Ana Cristina Duarte da Costa \\ Cristina Duarte ${ }^{1}$, Pablo Gordiano Alexandre Barbosa ${ }^{1}$, Laise Alves Candido Alves Candido ${ }^{1}$ \\ ${ }^{1}$ Instituto Federal do Ceará (IFCE) \\ $<$ amilcar.moreiraifce@gmail.com><crisduarte1@yahoo.com.br> \\ <pablogordiano@yahoo.com.br><laiseacandido@gmail.com> \\ DOI: <https://doi.org/10.21439/conexoes.v14i3.1465>
}

\begin{abstract}
Resumo. Se torna bastante perceptível nos dias atuais, a relação existente do ser humano com os animais domésticos com uma maior presença e influência destes, em cada lar. Assim exposto, a procura dos donos pelo bem-estar animal tem aquecido continuamente o mercado "pet" e com uma ampla gama de estabelecimentos comerciais que oferecem os mais diversos cuidados a estes animais. Essas crescentes demandas geram uma parcela significativa de resíduos sólidos que, por conta do grande volume e de suas características peculiares, merecem especial atenção quanto ao gerenciamento tanto interno quanto externo ao estabelecimento comercial. Neste sentido, este estudo objetivou diagnosticar a percepção de responsabilidade compartilhada quanto ao gerenciamento dos resíduos sólidos de serviços de saúde em estabelecimentos veterinários no município de Sobral - CE, utilizando a aplicação de questionário como mecanismo de levantamento de informação, bem como a visita in loco em alguns pontos pré-determinados. No estudo foi concluído que uma fiscalização mais ativa por parte dos órgãos reguladores poderia ser uma forma de incentivar os responsáveis desses estabelecimentos a cumprir as legislações vigentes, como o preconizado na Lei $n^{\circ}$ 12.305/2010 (Política Nacional dos Resíduos Sólidos) assim como foi demonstrado também, a inércia na pro atividade da população do município.
\end{abstract}

Palavras-chaves: Gerenciamento. Atividade Veterinária. Meio ambiente e Saúde Pública.

\section{AVALIAÇÃO DA PERCEPÇÃO QUANTO AO GERENCIAMENTO DOS RESÍDUOS SÓLIDOS EM ESTABELECIMENTOS VETERINÁRIOS DE SOBRAL/CE}

\begin{abstract}
It becomes quite noticeable in the present day, the existing relation of the human being with the domestic animals with a greater presence and influence of these, in each home. Thus, the demand of owners for animal welfare has continuously warmed the pet market and with a wide range of commercial establishments that offer the most diverse care to these animals. These growing demands generate a significant portion of solid waste that, due to the large volume and peculiar characteristics, deserve special attention regarding the management both internal and external to the commercial establishment. In this sense, this study aimed to diagnose the perception of shared responsibility regarding the management of solid waste of health services in veterinary establishments in the city of Sobral - CE, using the questionnaire as a mechanism for collecting information, as well a site visit at some predetermined points. In the study it was concluded that a more active inspection by the regulatory agencies could be a way to encourage the responsible of these establishments to comply with the current legislation, as recommended in Law $\mathrm{n}^{\mathrm{o}}$. 12.305/2010 (National Policy of Solid Waste), as demonstrated also, the inertia in the pro activity of the population of the municipality.
\end{abstract}

Keywords: Management. Veterinary Activity. Environment and Public Health.

\section{INTRODUÇÃO}

O aumento dos padrões de consumo e o acelerado crescimento populacional podem ser descritos como um dos maiores fatores responsáveis na geração de resíduos sólidos e que cresce numa à capacidade superior a taxa de absorção pela natureza e propiciando assim, diversos problemas ao meio ambiente (REIS et al., 2013).
Desta maneira, na medida em que o volume de resíduos aumenta constantemente, elevam-se os custos e surgem maiores dificuldades de áreas seguras para recebê-los. Com isso, faz-se necessária a minimização da geração, para que se atinja o desenvolvimento sustentável (BRASIL, 2006).

Parte importante dos Resíduos gerados pelas ativi- 
dades humanas são os relacionados a questão dos Serviços de Saúde - RSS, não pela quantidade produzida, pois é significativamente pequena em relação aos Resíduos Sólidos Urbanos - RSU, mas pelo potencial de risco que representam à saúde humana e ambiental, merecendo especial atenção no seu gerenciamento (BRASIL, 2006).

Sabe-se através da Resolução n ${ }^{0}$ 306/04 da ANVISA e da Resolução n ${ }^{0}$ 358/05 do CONAMA que os RSS classificam-se em tanto com base em suas características intrínsecas, quanto aos riscos que podem acarretar ao meio ambiente e à saúde humana ou seja, : Grupo A - Infectantes (A1, A2, A3, A4 e A5); Grupo B - Químicos; Grupo C - Radioativos; Grupo D - Comuns e Grupo E - Perfuro cortantes ou Escarificantes.

Os resíduos oriundos da saúde animal seriam os provenientes da atividade de estabelecimentos veterinários e também descritos como enquadrados no grupo A, ou seja, os classificados por apresentarem a possível presença de agentes biológicos que, por suas características podem apresentam riscos de infecção (BRASIL, 2006).

No serviço de atendimento de animais são realizadas consultas, tratamentos clínico-cirúrgicos e internação nas clínicas veterinárias. São vários os tipos de resíduos produzidos por esses serviços, não necessariamente em quantidade, mas em variedade, podendo acarretar riscos de contaminação ao meio ambiente (PEREIRA, 2014).

Para Mangussi (2017), os resíduos gerados em estabelecimentos veterinários podem apresentar riscos potenciais, devendo os geradores se atentar ao descarte de seu lixo, pois além da responsabilidade social, as empresas são obrigadas por lei a dar um destino correto aos resíduos gerados por suas atividades.

Para Cunha (2013), os impactos que podem vir a ocorrer ao meio ambiente e a saúde humana pelo gerenciamento inadequado dos RSS das unidades hospitalares podem ser vários ou seja, desde elevados índices de infecção hospitalar até a incidência de epidemias e/ou endemias pelo consumo de água proveniente de lençóis freáticos contaminados.

Já para Oliveira (2010), o Plano de Gerenciamento de Resíduos Sólidos de Saúde - PGRSS dentro de um estabelecimento se apresenta como uma importante ferramenta de gestão para garantir a minimização dos custos e a garantia quanto ao atendimento das normas legais tanto para a saúde do trabalhador, como para o bem-estar da comunidade no entorno e do meio natural em geral.

Conforme Pereira (2014), todas as etapas do processo de gerenciamentos dos RSS são fundamentais e, conforme definido na Figura 1, deve contemplar todo o manejo, ou seja, da sua segregação até a disposição final.

Diante do exposto, o trabalho visa analisar a situação quanto ao gerenciamento dos RSS em estabelecimentos veterinários localizados no município de Sobral/CE, através de pesquisa, a fim de observar a percepção dos geradores dos estabelecimentos em relação aos impactos destes no meio ambiente.

\section{METODOLOGIA}

A cidade de Sobral encontra-se localizada na região Norte do Estado do Ceará nas coordenadas $03^{\circ} 41^{\prime} 10^{\prime \prime}$ de latitude sul e $40^{\circ} 20^{\prime} 59^{\prime \prime}$ de longitude oeste com altitude de 69 metros, possui uma área de 2.129,897 km2 e tem uma população de aproximadamente 205.529 habitantes (IBGE, 2017).

A pesquisa foi realizada em treze estabelecimentos veterinários localizados no município de Sobral- CE. O levantamento de dados ocorreu por meio da aplicação de um questionário "in loco", com foco na gestão interna dos resíduos, no sistema de gerenciamento dos RSS e, na percepção dos responsáveis dos estabelecimentos sobre os possíveis riscos do manejo inadequado. O conteúdo do questionário foi elaborado com base nas Resoluções no 306/2004 da Lei no 358/2005 do CONAMA."

\section{RESULTADOS E DISCUSSÃO}

Os dados coletados permitiram verificar as ações relacionadas à capacitação dos funcionários, a existência de um PGRSS, e a percepção dos responsáveis quanto suas responsabilidades para com o meio natural e a saúde humana mediante o gerenciamento adequado dos RSS.

Vale reforçar que todos os envolvidos que trabalham com RSS conforme disposto na Política Nacional de Resíduos Sólidos é importante um conhecimento mínimo dos riscos potenciais ao qual podem estar expostos devendo assim, os estabelecimentos realizar alguma prévia de capacitação tanto quanto ao manuseio quanto no gerenciamento dos mesmos.

Os resultados da pesquisa são mostrados na Figura 2 , onde $92 \%$ dos responsáveis pelos estabelecimentos veterinários responderam possuir conhecimento dos procedimentos adequados sobre o gerenciamento dos RSS, e também sabem dos impactos destes quando manejados e descartados incorretamente tanto para o meio natural quanto para a saúde pública.

Conforme o gráfico em questão, apenas $8 \%$ dos estabelecimentos disseram não possuir conhecimentos so- 


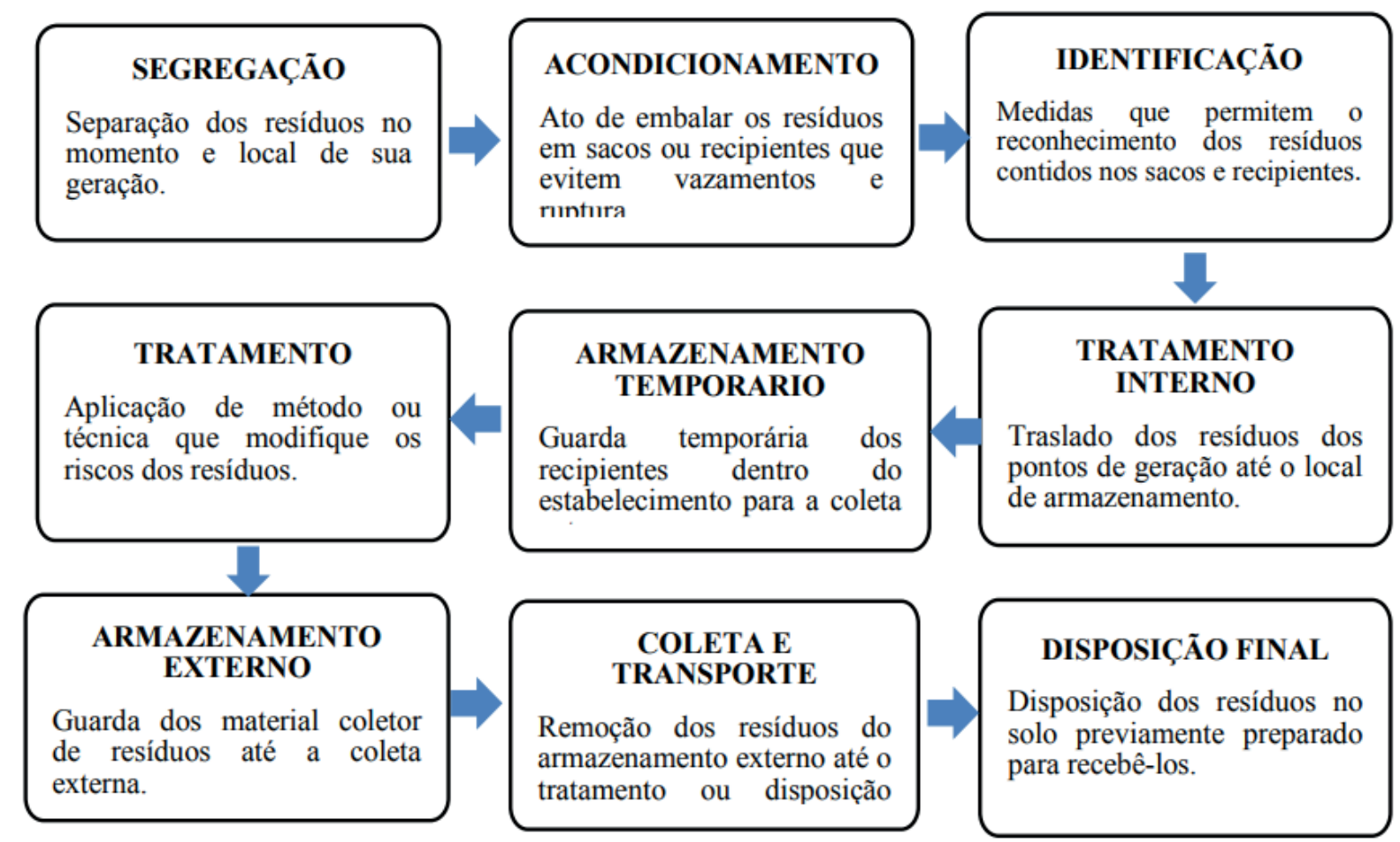

Figura 1: Fluxograma das etapas de manejo dos RSS

Fonte: Adaptado de Pereira (2014).

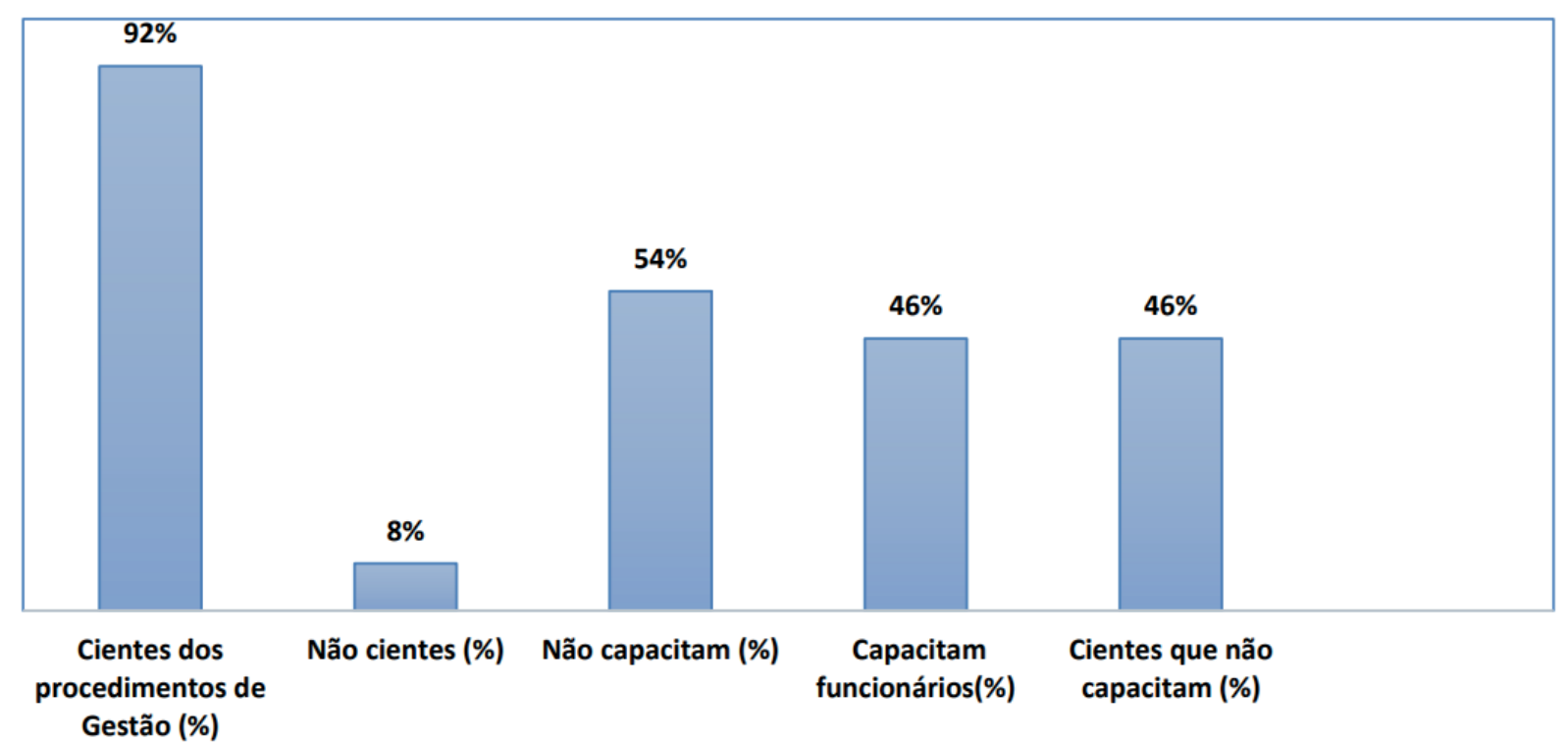

Figura 2: Diagnóstico dos estabelecimentos veterinários quanto à percepção do gerenciamento de RSS Fonte: autor. 
bre os procedimentos adequados de manejo dos RSS de acordo com o disposto na RDC nº306/2004 e na Resolução CONAMA nº358/2005.

Vale frisar a verificação de que apenas $46 \%$ dos estabelecimentos disseram orientar seus funcionários sobre as etapas de manejo dos RSS, e 54\% dos responsáveis disse não realizar nenhum tipo de capacitação quanto aos corretos procedimentos.

Todos os profissionais envolvidos diretamente ou não nas atividades de gerenciamento devem receber informações sobre o sistema adotado para o manuseio dos RSS, bem como norteamento em todos os procedimentos para as etapas de manejo dos resíduos sólidos, a fim de se evitar possíveis acidentes ocupacionais e contaminações, os quais podem se encontrar devidamente registrada em um documento como o PGRSS (BRASIL, 2006).

Todo o processo de manejo tem que ser gerenciado minuciosamente, com o intuito da proteção ambiental e social, quanto aos riscos inerentes que estes podem provocar (PILGER, 2008). Como mostrado na Figura 3, apenas $31 \%$ dos estabelecimentos veterinários localizados no município de Sobral/CE possuem um PGRSS.
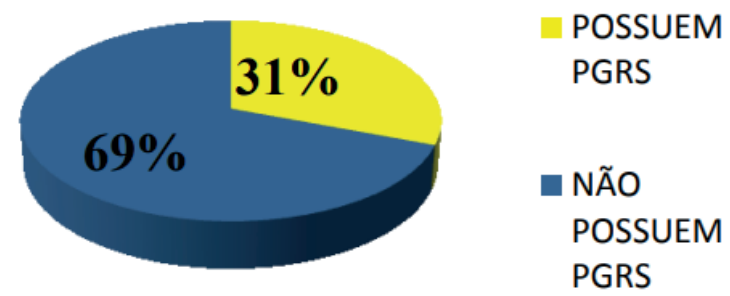

Figura 3: Estabelecimentos veterinários que possuem PGRSS Fonte: autor.

Vale ressaltar que, todos os estabelecimentos geradores de resíduos sólidos devem elaborar um documento contendo todas as etapas do manejo e ações de proteção para a saúde pública e para o meio ambiente, sendo destacado tanto na RDC n ${ }^{\circ} 306 / 04$ da ANVISA quanto no CONAMA n ${ }^{\circ} 358 / 05$ a responsabilidade dos estabelecimentos de saúde na elaboração e implementação do PGRSS, de acordo com a legislação vigente (BRASIL, 2004).

A pesquisa também mostra que apenas 38\% dos estabelecimentos possuem uma empresa especializada para recolhimento e tratamento dos seus RSS considerados perigosos, e $62 \%$ dos estabelecimentos entrevistados enviam seus resíduos para o aterro sanitário, sendo recolhidos pela coleta municipal da prefeitura (Figura 4). É importante salientar que, o destino final dos RSS, bem como a minimização dos impactos nega- tivos no meio ambiente, requerem alguns cuidados nos processos de tratamento (CALEGARE et al., 2006).

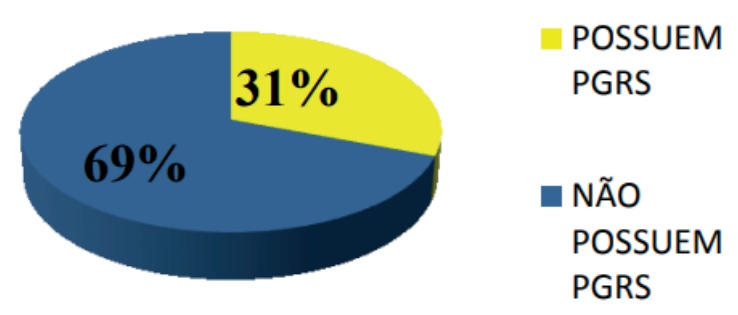

Figura 4: Estabelecimentos Veterinários que possuem empresa especializada para coleta e destinação final dos RSS.

Fonte: autor.

\section{Considerações finais}

A partir da análise dos dados obtidos na presente pesquisa, conclui-se que os estabelecimentos veterinários do município necessitam de maior atenção e fiscalização por parte das autoridades de saúde e ambiental, quanto dos riscos que os RSS acarretam ao meio ambiente assim é necessária a adoção de medidas de ação e programas de educação ambiental, para a orientação aos proprietários e responsáveis quanto a sua responsabilidade de gerador.

A maioria dos estabelecimentos não possui um PGRSS, evidenciando o descanso em relação aos possíveis riscos inerentes ao gerenciamento inadequado dos RSS. A implantação de um PGRSS é um aspecto fundamental para o desenvolvimento sustentável, pois segundo Calegare et al. (2006), os benefícios do controle da poluição é algo que atinge a sociedade em toda a sua extensão.

Observou-se que os responsáveis dos estabelecimentos possuem conhecimento quanto aos procedimentos adequados de gerenciamento dos RSS e os impactos negativos que os mesmos contrariando as normas legais podem acarretar ao meio. No entanto, infelizmente, ficou perceptível na pesquisa, por vezes, o foco dos estabelecimentos veterinários está na questão de somente dispor de serviços à população, como comércio e a geração de renda, isentando desta forma, os possíveis danos quanto às questões sanitárias e ambientais.

Diante do exposto, tornam-se imprescindíveis ações de fiscalização por parte do município, de forma a gerar um despertar nos responsáveis dos estabelecimentos quanto ao maior interesse em se adequar as normas da legislação vigente e contribuindo assim, para na proteção dos trabalhadores, na preservação da saúde pública e ambiental bem como, em uma melhor da qualidade de 
vida para a sociedade.

\section{REFERÊNCIAS}

BRASIL. Resolução RDC n⿳ 306, de 07 de dezembro de 2004. Dispõe sobre o Regulamento Técnico para o gerenciamento de resíduos de serviços de saúde. 2004. Diário Oficial da União. Disponível em $<$ http://www.unesp.br/pgr/pdf/rdc30604anvisa.pdf>.

Manual de gerenciamento de resíduos de serviços de saúde. 2006. Disponível em: $<$ http://www.anvisa.gov.br/servicosaude/manuais/ manual_gerenciamento_residuos.pdf $>$.

CALEGARE, L.; CARGNIN, M. T.; RUPPENTHAL, J. E.; SILVEIRA, D. D. Gerenciamento ecologicamente correto de resíduos de serviços de saúde: Um estudo de caso. In: XIII SIMPEP. Bauru, SP: Simpósio de Engenharia de Produção, 2006. v. 1, n. 1, p. 1-12. Disponível em: <http://www.simpep.feb.unesp.br/ anais/anais_13/artigos/517.pdf $>$.

CUNHA, G. F. Gestão de Resíduos de Serviços de Saúde: Estudo Comparativo em Unidades Hospitalares de Aracajú/SE. Dissertação (Mestrado) - Universidade Federal de Sergipe, São Cristóvão, 2013.

IBGE. Censo demográfico do município Sobral- CE. Brasil, 2017.

MANGUSSI, J. Lixo do pet shop e clínica veterinária: como descarta-lo corretamente. Revista Pet Center. 2017. Disponível em: <http:// www.revistapetcenter.com.br/materias/ler-materia/197/ lixo-do-pet-shop-e-clinica-veterinaria-como-descartal -lo-corretamente-aprenda>.

OLIVEIRA, E. C. Análise do gerenciamento dos resíduos sólidos de serviços de saúde: $\mathrm{O}$ caso de um hospital de médio porte do interior do estado de são paulo. Periódico Eletrônico Fórum Ambiental da Alta Paulista, v. 6, n. 12, p. 782-799, 2010.

PEREIRA, C. D. S. Diagnóstico do gerenciamento dos Resíduos Sólidos de Serviços de Saúde nos Estabelecimentos Veterinários de Mossoró-RN. Dissertação (Mestrado) — Universidade Federal Rural do Semi-Árido - UFERSA, Mossoró, 2014.

PILGER, R. R.; SCHENATO, F. Classificação dos resíduos de serviços de saúde de um hospital veterinário. Engenharia Sanitária e Ambiental, SciELO Brasil, v. 13, n. 1, p. 23-28, 2008.
REIS, M. A.; RANGEL-S, M. L.; MATTOS, C. M. d.; FRANKE, C. R. Conhecimento, prática e percepção sobre o gerenciamento de resíduos de serviços de saúde em estabelecimentos médicos veterinários de salvador, bahia. Revista Brasileira de Saúde e Produção Animal, v. 14, n. 2, p. 287-298, 2013. 